CASE XXxiII. - Right ovary; antiseptic method; two ligatures soaked in carbolised oil. Much trouble with bowels commencing before they had acted, afterwards threatening complete obstruction; gradually passed off ; and patient went out on twenty-fourth day.

CASE XXXIV (D).-Left ovary; antiseptic method; two strong silk ligatures soaked in watery solution of carbolic acid ( $\mathrm{I}$ in 20 ); three fine ones in carbolised oil. No after-trouble. Went home on nineteenth day.

CASE XXXV.-Right ovary ; antiseptic method ; two strong silk ligatures soaked in watery solution of carbolic acid ( $I$ in 2O); partial flap operation mentioned above. No after-trouble. Bowels open on eighth day. Patient up on couch on eleventh day.

CASE XXXVI.-Right ovary; antiseptic method; five medium silk ligatures soaked in watery solution of carbolic acid; partial flap operation. No after-trouble. Went out well on seventeenth day.

CASE XXXVII-Left ovary; antiseptic method; four medium silk ligatures (carbolised). No after-trouble.

CASE XXXVIII.-Left ovary; antiseptic method; two fine silk ligatures (carbolised); partial flap operation. Went out quite well on nineteenth day.

In the above cases, I have placed the letter (D) to those in which drainage was employed, as it is often an important element of success in cases treated by ligature. Other details I have purposely left out, as they are such as are common to cases treated by any method, and therefore do not affect the special question of ligature.

I may mention that I have followed the history of all my cases since they left my care, and in no case have I heard of any after-mischief from the ligature. All upon whom I have operated, who recovered, were still in good health a few weeks ago.

\section{ON THE THERAPEUTIC USE OF IODOFORM.}

By BERKELEY HILL, M.B., F.R.C.S.,

Professor of Clinical Surgery in University College; Surgeon to University College Hospital, and to the Lock Hospital.

LOCALLY, iodoform, as a dry powder, brushed lightly over the surface with a moistened camel-hair pencil, has been for three years my almost invariable treatment of venereal sores, especially the local chancre. During the last few months, I have often substituted for the dry powder an ethereal solution (one part of iodoform in six or eight of ether). The sore is touched or dabbed with a pencil dipped in the ethereal solution, according to its size and depth, lightly or copiously. The ether quickly evaporates, leaving a thin pellicle of iodoform, that as effectually stays the spread and produces healing of chancres as does the more copiously applied dry powder. Thus the surface is covered more exactly, and the disagreeable smell of the iodoform is too faint to attract attention. The sore is well washed with water and dried before the iodoform is applied, and the surface is lastly protected by a bit of dry lint. When the secretion is abundant, the dressing must be renewed twice daily, but in three or four days the amount of discharge becomes so scant that one dressing per diem suffices.

In this way, venereal sores heal quickly. Pain subsides at once; the sore is well in a week or ten days, and the chances of consecutive inoculation or bubo are greatly lessened. In a very few cases, the application of iodoform gives momentary smarting, which is very bearable ; even the ethereal solution does not hurt, and usually the patient declares the application to be quite painless. I avoid using iodoform on inflamed sores, or on simple granulating wounds; but indolent nonspecific ulcers are rapidly improved by iodoform locally applied.

Lately, I have given iodoform internally with great benefit. It acts more rapidly than potassic or other iodides, and, judging from experience thus far, is as readily borne as are those salts. I have given it in one-and-a-half-grain doses as a pill with extract of gentian. Three pills are given each dav, increasing gradually till eight or ten pills are taken in twenty-four hours.

I have used it with excellent effect in cases of obstinate syphilitic ulceration of the rongue, where the dorsum is covered with rugged thickened epithelium, which is constantly splitting into deep fissures, and thus causing continual severe pain to the patient. This affection is often quite insensible to mercury, alkaline iodides, or arsenic-the remedies usually beneficial. In three of these obstinate cases, where I had been treating the patients at intervals for years with the remedies just mentioned with little lasting benefit, iodoform-pills have acted like a chaım. Pain, immediately lessened, in two or three days ceased wholly; and the fissures healed rapidly, while the tongue soon shrank to its natural size. How lony the relief will endure, time alone will show ; but any interval of only apparent cure of this very painful affection is a great blessing to the sufferer, and time is given for the exhibi- tion of mercury if required. In December last, I had under my care in University College Hospital a patient with ulcerated and protruding gumma of the left testis, non-ulcerating gumma of the right testis, and ulcerating gummata of the skin over the upper end of the right tibia, with other syphilitic affections. Iodoform was administered in pills, and water-dressing applied to the ulcers. Rapid healing and subsidence of the swellings took place, notwithstanding that, when the dose of eight pills per diem had been reached and administered for three days, an outbreak of pyrexia, coryza, and iodic acne rendered it necessary to drop the drug completely for a short time. In three weeks, the patient left the hospital almost healed, and continued his treatment as an out-patient. Again, a lady who has during the last two years consulted me occasionally for intensely agonising pain in the head caused by syphilitic pericranial and cranial disease, for which a customary dose was thirty grains of sodium iodide three times daily, was at once relieved of pain by the iodoform pill taken three times daily, though, on the third day, nausea became too urgent to allow the iodoform to be continued in that quantity; it was at first diminished till pain ceased, and then discontinued altogether. This small experience has satisfied me that in iodoform we have a very useful addition to our store of weapons for fighting syphilis. Further observation will enable us to apply it more exactly and when most suitable.

\section{THERAPEUTIC MEMORANDA.}

\section{CHLORAL-HYDRATE IN DELIRIUM TREMENS.}

A sHORT time ago, I was almost despairing of a case of delirium tremens. The man was most violent, and in a fearful state of excitement ; and the remedies adopted appeared only to increase his activity and make him more and more unmanageable. The treatment had been Battley's solution in half-drachm doses; afterwards pure solution of the hydrochlorate of morphia by subcutaneous injection, as much as one grain repeated every two hours. There was no vomit. ing of the mixtures given on any occasion; these being, in addition to the liquor opii sedativus just mentioned, half-drachm doses of tincture of digitalis given every two hours, etc. After two or three days of the above treatment, and no improvement taking place, I determined to try the chloral-hydrate. Accordingly, at 5. IO A. M. I gave him half a drachm (thirty grains), and the same quantity at 5.40. At 6.0 , he had a subcutaneous injection of half a grain of morphia. At 6. Io, forty grains of chloral were given; at 6.25 , two-thirds of a grain of morphia were injected; and at 6.45 he was asleep. The man slept for eight hours, and awoke without headache or other unpleasant feeling except great thirst. He was now supplied with good nourishing food (beef-tea, etc.), and he was out walking next day. The quantity of the chloral given was one hundred grains, and of morphia one grain and one-sixth, in the space of an hour and fifteen minutes. Previously to the administration of chloral, the pupils were contracted to a point : an indication, of course, that the previous mixtures had been absorbed, but, as we have seen, with the effect only of increasing the excitement. Considering that the preparations of opium given previously had not conduced to somnolency, I attribute this condition to the chloral-hydrate chiefly, if not entirely. In another obstinate case of delirium tremens, in which the usual treatment by digitalis, morphia, etc., was ineffectual, I had recourse to chloral, repeated every ten minutes till one hundred and sixty grains had been taken. The patient then fell over, and, after sleeping for seven hours, was, on awaking, altogether a changed man.

I may add that, during the first two doses, there is always increased excitement, the patient becoming very garrulous -indeed, intoxicated, to all appearance ; but this soon gives place to thick speech, inarticulate mumblings, and peaceful sleep.

J. FARRAR, L.R.C.P.Ed., etc., Morecambe, Lancaster.

\section{PILOCARPIN, THE ACTIVE PRINCIPLE OF JABORANDI.}

A MORE elegant and effective way of introducing the active properties of jaborandi into the system than that proposed by Dr. Coghill as a therapeutical remedy in hydrophobia (vide BRITISH MEDICAL JOURNAL of January 5 th) would be by the hypodermic injection of the a!kaloid named by its discoverer, Merck, pilocarpium muriaticum (muriate of pilocarpine). It consists of transparent white crystals, and forms an easy and clear solution in equal parts of water. A hydrochloric solution of two per cent. is used for subcutaneous injection, one gramme or sixteen minims of which are equivalent to five grummes or eighty minims of the infusion of jaborandi-leaves. About one to one- 